Revista Mexicana de Astronomia y Astrofifica, Volumen 3, Mayo 1977

\title{
THE DISCOVERY AND OBSERVATION OF MULTIPLE STELLAR SYSTEMS
}

(Invited Paper)

A. H. Batten

Dominion Astrophysical Observatory, Victoria, B. C. Herzberg Institute of Astrophysics

AND

C. D. Saarfe

Department of Physics, University of Victoria, B. C.

\section{RESUMEN}

Se ha hecho una reseña de las dificultades que se presentan en el descubrimiento y observación de sistemas estelares múltiples, con especial énfasis en aquellos sistemas que se observan mejor mediante una combinación de métodos visuales y espectroscópicos, o bien, visuales y fotométricos. Se destacan las dificultades que existen a menudo en el proceso de hacer compatibles los resultados de los dos diferentes métodos de observación. Se incluye también una breve discusión de la posibilidad de detectar espectroscópicamente compañeras invisibles de poca masa.

\section{ABSTRACT}

The difficulties of discovering and observing multiple stellar systems are reviewed and discussed with especial reference to those systems which are best observed by a combination of visual and spectroscopic or visual and photometric means. Attention is drawn to the difficulties that often exist in reconciling the results of two different methods of observation. A brief discussion of the possibility of detecting spectroscopically invisible companions of low mass is included in the paper.

\section{INTRODUGTION}

Although a number of stellar systems are known whose multiplicity has been detected entirely by visual means they usually consist of a third star at a considerable distance from a much closer pair or of a similar but more complex arrangement of more than three stars. Only in a few cases has the mutual revolution of the close pair and the distant companion (for example) been detected. More often the physical connection is manifested only by a common proper motion. No doubt closer systems with two or more perceptible orbital motions are more interesting because more can be learned from them; they are, however, more difficult both to discover and interpret. Spectroscopic observation immediately suggests itself as a means of studying such closer multiples, but in fact very few of them have been discovered by this kind of observation alone. Even spectroscopic binaries are sometimes difficult to detect, as is shown by the controversies that surround estimates of the frequency of spectroscopic binaries (Batten 1973). The presence of more than two bodies in a system complicates the observed motions and may also produce a spectrum very difficult to measure and interpret. Evans (1968) has introduced the useful concept of hierarchy to classify multiple systems. This is illustrated in Figure 1 which shows diagrammatically two quadruple systems of different hierarchies. Case (i) could 
well be a wide (visual) pair, each component of which is spectroscopically double; several known quadruples are of this type. One could imagine a system, however, in which none of the components are visually resolvablc; in this case, the multiplicity assigned to the system would depend on the number of separate spectra visible, that is to say on the relative luminositics of the components. If only one spectrum were visible, or if only stars $a$ and $b$ or $c$ and $\mathrm{d}$ had visible spectra, we should at most recog-

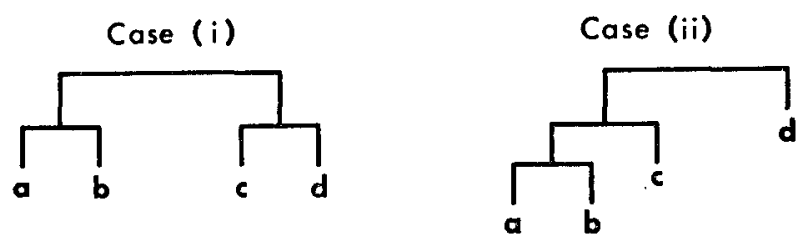

FIg. 1. Two quadruple systems of different hierarchies (after Evans).

nize the system to be triple. Even if three or even four spectra were all visible, the resulting pattern of lines might be so complex (depending on the spectral types) that we would fail to recognize the full degree of multiplicity. Case (ii) presents other problems. If either $a$ or $b$ gave the only visible spectrum, then d's presence would be very hard to dctect. If $c$ were the bright component, the system might be thought to be triple, ab being regarded as a single faint component, while if $d$ had the one visible spectrum the system would be thought to be merely double. In both cases, from spectroscopic observations alone we would be very likely to underestimate the true multiplicity of the system. In fact all stellar systems known to contain more than three stars have at least one visually resolvable component.

For these reasons we shall confine our attention in this review primarily to systems whose multiplicity is revealed by the combination of two or more types of observation - in particular spectroscopic and visual, or photometric and visual (cases in which a multiple system contains an eclipsing binary). As a special case of the use of spectroscopic observations we shall consider their application to the detection of invisible components of low mass. Finally we shall discuss the problems of reconciling the results of different methods of observation.

\section{SPECTROSCOPIC AND VISUAL OBSERVATIONS}

In what follows we shall be almost entirely concerned with triple systems; after "simple" binaries they are both the most numerous and the easiest to interpret. We have alrcady indicated in the previous section some of the difficulties of drawing conclusions about systems of higher multiplicity. We have also pointed out how the interpretation of a system may be affected by the number of spectra that are visible, and we will consider the various possibilities in turn.

(i) Three spectra; all similar: These are the most interesting of triple systems since they offer the possibility, when both kinds of observation are combined, of determining the system completely. Most known systems of this type are of the spectroscopicvisual kind. That means that although they are resolved (as binaries) with an astrometric telescope and visual orbits exist for them, they appear as single stars on the spectrograph slit. Only the circumstance that one of the visual components is a spectroscopic binary enables us to study the system spectroscopically at all, because the velocity difference in the visual orbit would usually be too small for the spectra to be resolved if both the visual components were single stars. Known systcms of this type range in spectral type from late $A$ to $G$. Evans has pointed out that this may be a selection effect since in spectra of earlier types the lincs are often too broad to be resolved into their components, and in those of later type the lines are so crowded that the displaced spectral lines of one star can be superposed on those of another. Thus HD 100018 (ADS 8189) with three spectra of early $F$ type (Petric and Batten 1969) is one of the more favourable examples of this class; its triple nature would eventually have been found by high-dispersion spectroscopy even if the visual companion were not resolvable. On the other hand HD 165590 (ADS 11060) is much harder to interpret. Its three spectra are all of class $G$, and only the fact that it is a known visual binary makes us certain that it is triple. Eventually the combination of the spectroscopic and visual observations of the long-period orbit should enable us to determine the inclination of that orbit and the total mass of the system. Then, since the mass of the spectroscopic binary is known, the 


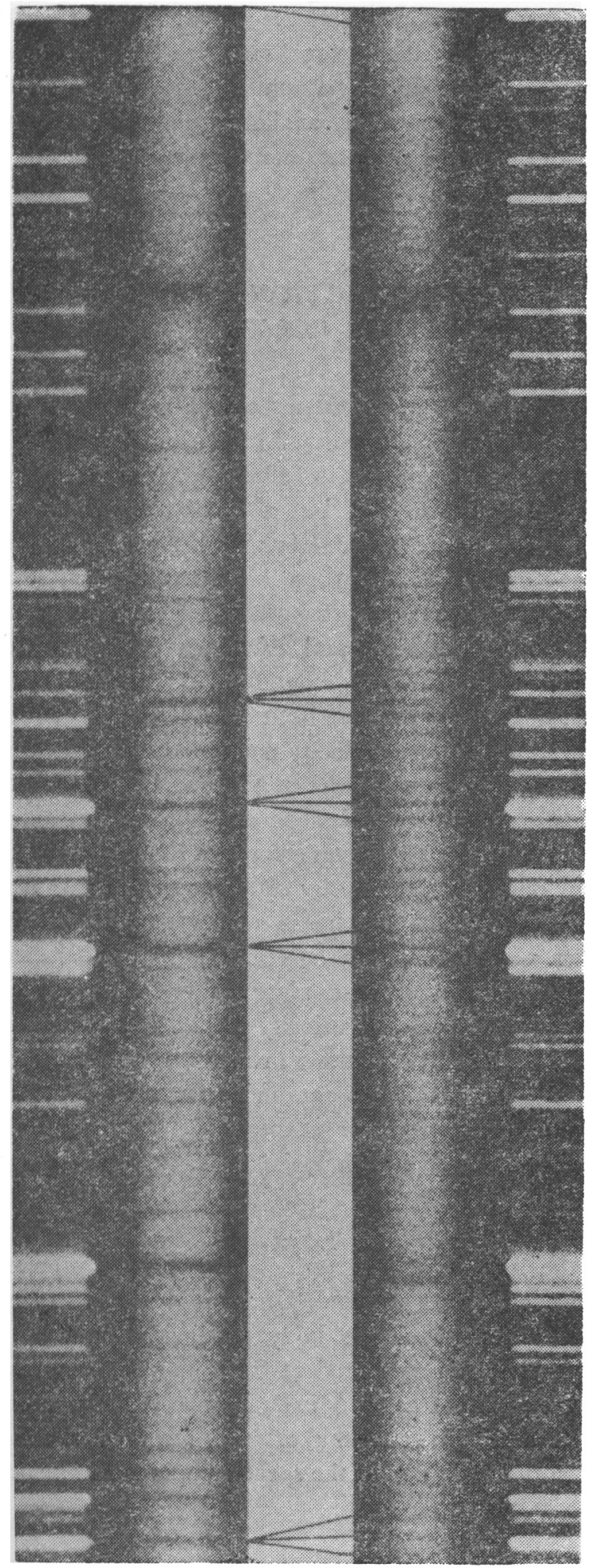

Fio. 2. A portion of the spectrum of ADS 8189 at singleline (left) and triple-line phases. masses of each of its components, and the inclination of its orbit can be determined. Such investigations are necessarily long-term projects, however, and attempts of this kind have encountered difficulties, such as those described by Petrie and Batten in the case of ADS 8189, which will be discussed in a later section. In extreme cases, as Evans has pointed out, we may be uncertain whether or not the system contains more than three components.

(ii) Three Dissimilar Spectra: This case is apparently less common, but a few examples are known. A good one is 59 Serpentis which consists of two A-type stars in a short-period orbit with a brighter G-type giant companion whose orbital period is close to a year. The spectrum is a composite one in which the early-type component is periodically seen to double. In some respects these systems are easier to deal with, but since the A-type components have only a few spectral lines (which are usually broad) their velocities are determined with only a rather low accuracy. Most known systems of type (i) contain three main-sequence stars. Systems of this type, however, must contain at least one evolved star, and this perhaps helps to account for their relative rarity. Two systems of this kind (59 Serpentis and HD 157978/9) have been studied spectroscopically but in neither case is the long perind observable visually.

(iii) Two Spectra Visible: If the invisible spectrum is that of one of the stars in the short-period orbit, the triple nature of the system is easily recognized by the rapid variation of the velocity of the other component in that orbit. An example is ADS 14893 recently studied by West (1976). The two visible stars are of approximately equal magnitude and (late F) spectral type. One of them, however, shows a radial-velocity variation in a period of just over 2.2 days. Incidentally, this system provides a good example of the interaction of visual and spectroscopic observations. The separation is always small $\left(\leq 0^{\prime \prime} .25\right)$ and the nearly equal brightness of the two stars has led to confusion of the quadrant. West has shown that the long period is almost certainly half the period of about 12 years found by Baize (1959) from visual observations alone.

If the invisible component were the distant companion, the slow variation of the centre of mass of the close pair could be detected although to do 
so is usually more difficult than in the case just considered. Of course, in this situation, the system is probably not recognized as a visual binary.

(iv) Only One Spectrum Visible: We must again distinguish between the case in which the single spectrum is that of one of the close pair, or that of the distant companion. The second of these, however is largely academic since such a system would not be recognized as a triple at all and might even be overlooked as a binary. For example, if the two A-type components of 59 Serpentis were only a little fainter, their spectra would not be seen in the combined light of the system and the single K-type star might not have attracted enough attention for its variable velocity to have been discovered.

If the one visible spectrum belongs to a member of the close pair the triple nature of the system may be deduced from variations of the systemic velocity, $V_{0}$, with time. These are often hard to establish certainly; a good example may be HD 129132 (Harper and Blanchet 1937), and others are b Persei (Hill et. al. 1976) and $\lambda$ Tauri which is well known. Other orbital elements may also appear to vary, if the two periods are comparable. This was shown in the case of Algol by McLaughlin (1934) and discussed more generally by Petrie and Batten (1965). The systems mentioned so far have no visually resolvable components -although a photocentric orbit exists for Algol (van de Kamp et al. 1950)-but there are spectroscopic-visual triples showing only one spectrum, e.g. $\mu$ Orionis, in which the visual secondary and the spectroscopic companion are both too faint to show up in the combined light. Without the knowledge of the visual orbit the triple nature of single spectrum systems is often hard to detect because the tell-tale variations in the orbital elements are small and often near the limits of significance. Without other lines of evidence (the change of spectrum during eclipse and the light-time effect on times of minima) we might still question the triple nature of Algol, as we do that of AR Cassiopeiae whose orbital elements also vary in an unexplained manner.

Perhaps the principal conclusion to be drawn from this survey of the various classes of triple system is that selection effects that are hard to quantify are likely to lead us to underestimate both the numbers of multiples and the degree of multiplicity. Nevertheless, systematic reobservation of many spectroscopic systems for which observations at only one epoch are currently available might well lead to the discovery of at least more triples.

\section{DETECTION OF LOW MASS COMPANIONS}

A special group of systems containing at least one invisible component is that in which a body of substellar mass is believed to be present. Not all systems like this are multiple, but a number of them are (at least if the existence of the low-mass component is confirmed) and as there has recently been some argument about the astrometric evidence for some of these (Gatewood and Eichhorn 1973; van de Kamp 1975) this may be a good opportunity to discuss the contribution that can be made to the discussion by radial-velocity determinations. The best spectrograph for this work at Victoria is at the coudé focus of the $1.2 \mathrm{~m}$ telescope and gives a dispersion of $2.4 \AA \mathrm{mm}^{-1}$. The mean error of a single determination of the radial velocity of a star can be less than $0.2 \mathrm{~km} \mathrm{~s}^{-1}$, if the star has a late-type spectrum with many well defined lines. (This is the external error determined from plate-to-plate scatter.) Thus we can expect to determine a velocity variation of $0.5 \mathrm{~km} \mathrm{~s}^{-1}$ fairly easily, and sometimes one even smaller. On the other hand the spectrograph is limited to objects brighter than sixth, or at best seventh, magnitude. Thus Barnard's star is quite unobscrvable. Let us, however, consider it as an example. In his latest discussion, van de Kamp derives two perturbations in the motion of the star, one of an $11.5 \mathrm{y}$ period and one of a $22 \mathrm{y}$ period. Under reasonable assumptions the mass of the body producing the $11.5 \mathrm{y}$ perturbation is estimated at $0.001 \mathrm{~m}_{\odot}$ and the mass of the primary as $0.15 m_{\odot}$. The orbital inclination is close to $90^{\circ}$ and the orbit is assumed circular. From the definition of the spectroscopic mass function

$$
\begin{aligned}
f(m) & \equiv \frac{m_{2}^{3} \sin ^{3} i}{\left(m_{1}+m_{2}\right)^{2}} \\
& =1.0385 \times 10^{-7}\left(1-e^{2}\right)^{3 / 2} K_{1}^{3} \mathrm{P}
\end{aligned}
$$


(where $\mathrm{K}_{1}$ is in $\mathrm{km} \mathrm{s}^{-1}, \mathrm{P}$ in days, and $\mathrm{m}$ in solar masses) we can easily see that

$$
\frac{0.963 \times 10^{7}}{\left(1-\mathrm{e}^{2}\right)^{3 / 2}} \frac{\mathrm{f}(\mathrm{m})}{\mathrm{P}}=\mathrm{K}_{1}^{3}
$$

Thus for Barnard's star $\mathrm{K}_{1}$, which is half the expected total velocity range of the primary star, has the value $0.05 \mathrm{~km} \mathrm{~s}^{-1}$, approximately. Even if the stars were bright enough to observe, we could not hope to detect the velocity variation unless we were prepared to obtain and measure twenty to thirty plates a year. If we set $K_{1}=0.25 \mathrm{~km} \mathrm{~s}^{-1}$ as the minimum variation we can detect from measures of only a few plates, we find that

$$
\frac{0.963 \times 10^{7} \mathrm{f}(\mathrm{m})}{\left(1-\mathrm{e}^{2}\right)^{3 / 2} \mathrm{P}} \geq 0.015
$$

for a low-mass invisible companion to be detected from its radial velocity variation. This means that for a circular orbit inclined at $90^{\circ}$ to the plane of the sky

$$
\mathrm{f}(\mathrm{m}) \geq 1.66 \times 10^{-0} \mathrm{P} \text {. }
$$

If $\mathrm{P}$ is of the order of ten years, and the primary star has a mass equal to the Sun, one would expect to be able to detect the radial-velocity variation produced by a dark companion of about $0.02 m_{\odot}$. There are several systems suspected containing dark companions with masses in the range $0.01 m_{\odot}$ to $0.1 m_{\odot}$ and three -61 Cygni, 70 Ophiuchi, and $\xi$ Bootis- are under study at Victoria. If the existence of these bodies is confirmed the systems are triple. The evidence for two of them is discussed below: observations of $\xi$ Bootis are not yet reduced.

The two stellar components of 61 Cygni have an orbital period estimated at over 650 years. Strand (1957) and Deutsch (1958) have both published astrometric evidence for an invisible companion to star A with an orbital period close to 5 years. Van de Kamp (1973) has also published an astrometric study. Radial velocities have now been obtained over one five-year cycle by J. M. Fletcher. Apart from two anomalous observations near the beginning of his series, there is no cvidence of any variation although the estimated mass of the companion is $0.01 m_{\odot}$ and the expected velocity range of 61 Cygni $\mathrm{A}$ is about $0.4 \mathrm{~km} \mathrm{~s}^{-1}$.
In 70 Ophiuchi the opposite situation obtains. It has been observed at Victoria since 1966, and since 1970 with the $2.4 \AA \mathrm{mm}^{-1}$ spectrograph. The radial velocity of the primary component was also observed for many years at the Lick Observatory (Berman 1932) and showed some evidence of a seventeenyear oscillation imposed on the radial-velocity variation produced by the 88-year orbital motion of the pair AB. Early astrometric observations appeared to support the spectroscopic evidence for a third body, but Worth and Heintz (1974) believe that those observations contained large errors of measurement made by inexperienced observers. The radial velocities may also be affected by relatively large errors, and the high-precision observations must be continued for some time before we can be sure whether departures from the expected orbital motion of $\mathrm{AB}$ are real or not. Batten and van Dessel (1976) still find some marginal evidence for a fifteen-year perturbation which could be produced by a dark companion to A of mass about $0.05 \mathrm{~m}_{\odot}$. They find a smaller amplitude for the perturbation than Berman did, but even so it is much too large to be reconciled with any existing astrometric data. This alone should make us very cautious about accepting the reality of the third body.

\section{VISUAL AND PHOTOMETRIG OBSERVATIONS}

Just as there are many visual binaries one (or both) of whose members is found to be a spectroscopic binary, there are several visual pairs which include an eclipsing binary as one member, or as both in the case of ADS 9537 (Batten and Hardie 1965) for example. Much of the above discussion is applicable to eclipsing systems. However it should be borne in mind that the known eclipsing binaries are on the average much fainter, and therefore probably more distant, than are the known noneclipsing spectroscopic systems. This effect has three consequences.

1. Most of the visual pairs which include an eclipsing system are of such long period that little if any orbital motion can be detected either astrometrically or spectroscopically. 
2. It will be less easy in general to resolve visually any third bodies discovered by purely photometric means than to obtain visual resolution of spectroscopic triples.

3. High-dispersion radial velocities are difficult to obtain for eclipsing systems.

As a result, one may expect to be restricted to purely photometric studies of a triple system more of ten than one is restricted to purely spectroscopic observations.

There are however three ways in which eclipsing systems lend themselves to the discovery and study of stellar multiplicity.

1. A faint third component may be seen or its spectrum detected during eclipse. This is in fact the way in which the triple nature of Algol was detected.

2. The presence of third light in the solution for the photometric elements.

3. The effect on the times of the eclipse minima of the light time in the long-period orbit.

Let us consider each of these effects in more detail.

The spectra of many eclipsing binaries are dominated out of eclipse by a single spectrum, with an additional spectrum being visible in eclipse, sometimes only during totality. Good spectrograms of the secondary spectrum are difficult to obtain since the systems are then often very faint, and only a limited time near mid-eclipse is available to obtain the spectrograms. If the spectrum is that of the secondary in the short-period orbit, the slope of its velocity. variation during eclipse may give a rough value of the mass ratio, as for example in U Cephei (Batten 1975). More often this slope is masked by observational errors, and by distortions of the spectrum possibly produced by gascous streams or shells. However, if the spectrum seen in eclipse is that of a third component, such a slope is not to be expected. Instead a slow velocity variation from eclipse to eclipse should occur, as it does in Algol (Ebbighausen 1958). Without a substantial collection of observations accumulated from many eclipses it may be impossible to decide whether the spectrum observed in eclipse is that of the eclipsing companion or of a third body. Perhaps this indicates, at least in part, why so few triples have been found in this way.
In similar fashion, to the authors' knowledge, no faint visual companions to eclipsing systems have been discovered by examining the system during eclipses, although it might be supposed that such a search would prove fruitful, especially for those systems with deep eclipses and faint companions, which are not readily detectable against the full light of the eclipsing system.

Many eclipsing binaries have visual companions close enough for their light to be difficult or impossible to eliminate by the diaphragm of a photometer. Their contribution to the total light must be determined and subtracted from that of the whole system before a photometric solution can be attempted for the eclipsing components. This is preferably done photoelectrically, and observations with an area scanner similar to that described by Franz (1971) might prove very useful for this purpose.

Conversely it might be supposed that for those systems too close for such a determination to be made, the light of the visual companion might be eliminated from the solution by trial and error as "third light", and that even closer systems, unresolved visually, might be found in a similar fashion. But such are the difficulties of photometric solutions that the size of the third component's contribution to the light of the system may vary from solution to solution, even for the same set of observations. In many cases too, a new and better set of observations has removed the necessity of invoking third light in many systems formerly believed to be triple on this basis. Indeed it sometimes seems that photometric observers have worked with the deliberate purpose of avoiding the use of third light! Even if third light is inevitable to obtain a solution, moreover it is not necessary to conclude that a third star is present. The light may be contributed by a gaseous stream or shell; indeed such circumstellar matter is thought to contribute the bulk of the light in certain old novae, e.g., DQ Herculis (Bath, Evans and Pringle 1974).

It therefore seems wise, before accepting third light as evidence for a triple system, to search for supporting evidence, in the form of a third spectrum visible perhaps only in eclipse, or of a variation of the systemic velocity, or of a light-time effect in the times of minima. Such searches as have been carried out, however, have frequently been unsuccessful, 
providing no confirmation of the existence of third stellar components, and in some cases giving upper limits to their light. One system in which a third light is supported by a third spectrum is V463 Cygni (Vetesnik 1968). The spectrum of a third component is also visible in DL Virginis (Schöffel and Popper 1974).

Times of minima of cclipsing binaries have bcen obtained for most of the past century, though unfortunately not uniil fairly recently from accurate photoelectric observations. Morcover often the observer is forced either by the weather, the duration of the eclipse, or the time and hour angle of mideclipse, to observe only a portion of any given eclipse, and by fitting pieces of light curves together to obtain a "normal minimum". This kind of result is inevitably of lower accuracy than a time of minimum derived from good coverage of a single eclipse, owing to the nccessity of making the rather risky assumption of the constancy of the light curve, and to the uncertainty in the epoch appropriate to the grouped cata. Hiowcver despitc these difficulties accurate photoclectric times of minima are now accumulating fairly rapidly, especially for the brighter systems, and it should soon be possible to detect the effect of light time in a long orbit.

This problem has been considered theoretically by Irwin (1952, 1959) who produced a set of standard light-time curves for various values of the eccentricity and longitude of periastron in the long orbits. In order to use these curves, however, it is necessary to know the correct mean cclipse period over a full cycle of the long orbit, and therefore at least two maxima or two minima of the light-time curve must be covered. In any case it is necessary to accumulate observations over this length of time in order to have confidence that the observed variation in the eclipsing period is a light-time effect, and even then one can be deceived.

From the observational point of view the problem has recently been addressed for several fairly wellobserved systems by Frieboes-Conde and Herczeg (1973), who discuss the observational difficulties that arise, among them the choice of the correct mean eclipse period mentioned above. More importantly the periodic effect sought may be masked by a series of random, rather abrupt, period changes, the nature of which is not understood, but which seem to occur in many systems. Frieboes-Conde and Herczeg also point out that times of minima of low accuracy are almost useless and tend to confuse the matter. In the opinion of the authors, for the purpose of detecting light-time effects, which are likely to be of low amplitude, all times of minima based on visual estimates should be scrupulously ignored for all systems except those with very deep short eclipses. Morcover all those minima derived other than by photoelectric observations of both descending and ascending branches of a single eclipse should be treated with the grea!cst rescrve. One has only to examine the $\mathrm{O}-\mathrm{C}$ diagrams for such systems as TV Cas, SV Cam, $\mathrm{U} \mathrm{CrB}$ or $\mathrm{U}$ Oph to realize the validity of this conscrvative view. Of ccurse for those systems with long eclipses or with inconvenient periods, times of minima derived from obscrvations of several cclipses are unavoidable. But since this kind of combination requires the assumption of a period and of the constancy of the shape of the light curve it should be avoided whenever possible.

Frieboss-Condc and Herczeg enumerate four criteria which any apparently periodic O.C diagram should satisfy before it is attributed definitely to the light-time effect. These are:

1. The O-C curve for primary minima should correspond to the line-of-sight component of motion in an elliptical orbit, i.e., it should conform to Irwin's curves.

2. The secondary minimum should show residuals similar to those for primary minimum, to eliminate apsidal rotation as a cause for the observed variation.

3. The mass function derived from the curve should not indicate a mass for the third component incompatible with its luminosity, for which at least an upper limit is always available.

4. A change in the systemic radial velocity of the eclipsing pair should be detected and should be compatible with the apparent light-time effect. Perhaps one should add that an astrometric orbit, compatible with the photometric one, would be a suitable alternative to the spectroscopic study, which as Frieboes-Conde and Herczeg point out, requires numerous high-quality radial velocities.

Frieboes-Conde and Herczeg use their criteria to assess the $\mathrm{O}-\mathrm{C}$ diagrams of 14 systems as candi- 
dates for light-time variations. None of the systems satisfy all of the criteria; indeed for many there is little evidence for any periodicities in the diagrams. For some, however, it is not possible to rule out a light-time effect, although none is confirmed.

Indeed to date, there is no case in which a wellestablished triple system has been discovered purely by photometric means. Even in the case of Algol the third component was first seen spectroscopically by Schlesinger, although it was not confirmed that the spectrum was that of the third component until an orbit was derived for it by Ebbighausen (1958). This orbit is at variance with that derived from the times of minima by Eggen (1948) but has been approximately reconciled with more recent minima by invoking an abrupt period change, according to Frieboes-Gonde, Herczeg and $\mathrm{H} \phi \mathrm{g}$ (1970). In the more recent case of VW Gephei (Hershey 1975), despite the fact that the variation of the times of minima prompted the astrometric search for a companion, the orbit derived from the astrometry predicts a light-time effect resembling that observed only remotely. Indeed as Hershey himself points out, the astrometric elements could not have been deduced from the times of minima.

In the case of QS Aquilae, Knipe (1971) has attributed an observed period change to motion in the visual orbit, whose elements are at present undetermined. Knipe's data are however scanty, include no continuous runs through a single eclipse, and cover only a fraction of the long period. Therefore a determination of the elements by comparison with Irwin's curves would be difficult and perhaps premature, and it was not attempted by Knipe.

Comments on two of the systems discussed by Frieboes-Conde and Herczeg are perhaps in order at this stage. RT Persei has been rediscussed by Mancuso and Milano (1975), who find that it is still possible to fit the data to a light-time curve, of which two full cycles have now been covered, without the necessity of introducing any abrupt period changes. They do however offer the alternative of no light-time effect but several abrupt changes. The light-time orbit satisfies fairly well the first three of Frieboes-Conde and Herczeg's criteria, but a decision between the two alternative interpretations will have to be based on a careful assessment of the fourth criterion. This will probably have to be ap- plied in its original spectroscopic form, despite the difficulty arising from the system's faintness, since for reasonable assumptions about the distance of RT Persei, the angular separation of the third component is predicted from the light-time effect to be not larger than 0.01 , which makes the alternative of an astrometric approach much more difficult than in the case of VW Cephei.

It is possible to add a little to the discussion of U Ophiuchi by Frieboes-Conde and Herczeg by considering photoelectric times of minima obtained since their paper was published. In addition to the three published by Scarfe et al. (1973) and one by Pohl and Kizilirmak (1974) three new photoelectric minima have been obtained at the University of Victoria. These were as usual, determined from observations in a single colour, reduced by the method of Kwee and van Woerden (1956), and are listed in Table 1. The values of $\mathrm{E}$ and $\mathrm{O}-\mathrm{C}$ in the table were calculated using the ephemeris of Frieboes-Conde and Herczeg.

TABLE 1

NEW MINIMA OF U OPHIUGHI

\begin{tabular}{cccl}
\hline JD 2440000+ & E & O-G (days) & \multicolumn{1}{c}{ Observer } \\
\hline $1387.7788 \pm 0.0003$ & 1557.5 & +0.0044 & C.D. Scarfe \\
$2552.8462 \pm 0.0005$ & 1954 & +0.0048 & D.J. Barlow \\
$2568.7818 \pm 0.0004$ & 1963.5 & +0.0057 & D.J. Barlow \\
\hline
\end{tabular}

$$
\begin{aligned}
& \text { Primary Minimum }=\text { Hel. JD } 2439275.3110+ \\
& \quad+1.6773441 \mathrm{E} .
\end{aligned}
$$

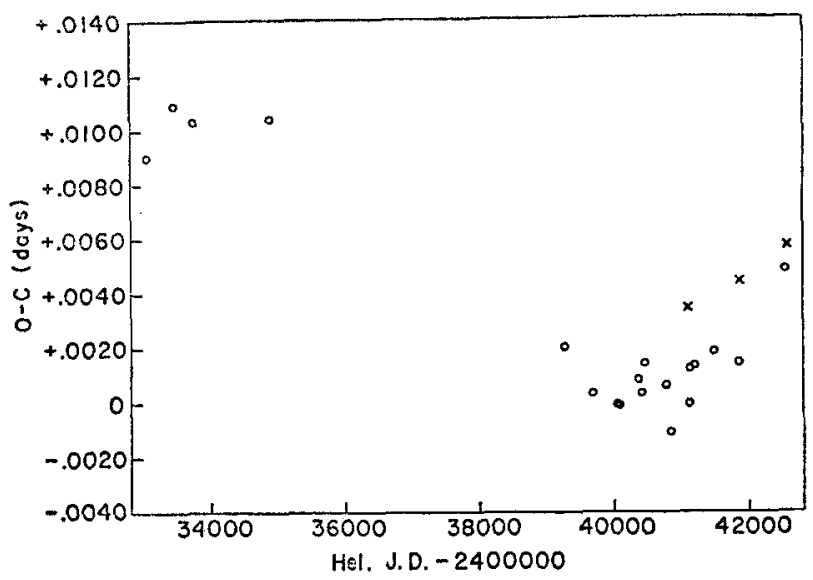

FIG. 3. (O-C) residuals of photoelectrically determined times of minima of $U$ Ophiuchi. o Primary Minimum. $x$ Secondary Minimum. 
All the photoelectric minima available to the authors are plotted in Figure 3. It is clear that the present trend is toward positive residuals, whereas both of the tentative light-time orbits suggested by FrieboesConde and Herczeg predict negative residuals at present, and can therefore be rejected. It is by no means clear that any light-time effect exists in this system, and many accurate photoelectric times of minima will be required in the future to confirm or deny its presence. The remarks of Duerbeck (1975) concerning accuracy of timings should be heeded in this connection.

It is perhaps worth noting that the residuals of secondary minimum, all obtained at the University of Victoria, seem to lie slightly above those of primary minimum. They thus confirm the conclusion of Eaton and Ward (1973) that the secondary minimum was displaced slightly from phase $0 .{ }^{\mathrm{P}} 5$, and show that this behaviour continued at least for a time after the epoch of their observations (JD 2440660). It is by no means certain that this displacement is permanent, or whether it is produced by a slight orbital eccentricity or by some small photometric perturbation.

In summary it is not easy to prove that an apparently periodic variation of the eclipse period is due to the light-time effect of a third body, nor is it easy to establish the existence of such an effect in the face of erratic period changes. Many of the more frequently observed eclipsing systems show such changes, unfortunately. However we should not conclude that the case is hopeless, since the periodic effect of apsidal motion is well documented in at least a few systems. For these systems the random changes are generally much smaller and less frequent than in Algol, for example. It is perhaps misleading therefore that Algol is a semidetached system and VW Cep a system of the WUMa type, believed widely to be contact binaries. Searches for the light-time effect of otherwise undetectable companions might be more profitable if restricted to detached unevolved systems.

\section{COMPARISON OF OBSERVATIONS MADE BY DIFFERENT MEANS}

A recurrent theme in this paper has been the differences between the results of observation of the same system using different methods. Thus difficulties have been encountered in reconciling the visual and spectroscopic observations of ADS 8189; two different periods have been found for ADS 14893 ; in 61 Cygni the astrometric observations indicate the existence of a third body and the spectroscopic ones do not, while in 70 Ophuichi the situation is reversed; finally the observed astrometric orbit of VW Cephei cannot be reconciled with the change of period of the eclipsing pair which first suggested the existence of a third body in the system.

There are several reasons for these discrepancies. Systems that can be observed both spectroscopically and visually are usually difficult to observe by either method. Even a system that a visual or astrometric observer will describe as "close" or "difficult" is likely to have a radial velocity that varies only slowly and so to be equally "difficult" for the spectroscopist. Systematic errors produced by blending of nearly equal spectra can also be important -especially in multiple systems. This has been discussed in some detail for ADS 8189 by Petrie and Batten (1969). The velocity difference between the centre of mass of the close pair and the visual secondary did not reach the value expected from the visual orbit at the time of periastron passage in 1967. One possible explanation -that periastron occurred a little later than predicted- can now be ruled out; plates obtained at a dispersion of $6.5 \AA \mathrm{mm}^{-1}$ in 1972 and 1973 indicate that the velocity of the secondary had by then already returned to its value of before the expected periastron passage. The source of the discrepancy is still unknown but may be discovered if observations are made at a still higher dispersion during the next periastron passage in 2053.

There must be similar sources of error acting in the systems 61 Cygni and 70 Ophiuchi. Unless there is some major innovation in technique, dark, low-mass companions will always be near the limit of detection both spectroscopically and astrometrically. The lesson to be learned from these systems is the importance of combining the two kinds of observation -if astrometric observations alone were available for 61 Cygni and spectroscopic ones alone for 70 Ophiuchi we might have no doubt about the dark companions - and of continuing both series of observations; more data may lead to the recon- 
ciliation that at present evades us. Meanwhile the conservative conclusion, that the existence of neither of these bodies has yet been demonstrated, is probably the safest.

By contrast there is no real problem connected with ADS 14893. The visual binary is of such a nature that we can well understand how the double value for the period came to be adopted. As in the care of other binary systems, the spectroscopic observations are able to remove ambiguities that are inherent in the visual observations. This example illustrates the general case that the behaviour of a binary normal to the plane of the sky cannot always be predicted from its behaviour in that plane itself. This again underlines the need for observing in both ways whenever possible. One should also try to deduce the orbit from both kinds of observation simultaneously. Hitherto we have had to take one sct of observations (usually the visual), deduce from them as much as possible, and then assume the resulting orbital clements in order to interpret the (often rather few) spectroscopic observations. Morbey (1975) has developed and described a method of converging to the orbital clements from both sets of data used together. This treatment is especially desirable when, as in many systems, radial velocities are so much the more useful near periastron and positional measurements are more useful in the rest of the orbit.

Detection of a triple system from variations of times of minima of an eclipsing pair may also involve pushing a technique to iis limit. The light-time fluctuation in the long-period orbit of Algol is now well established and the pcriodic variation in times of light minima of the eclipsing pair has an amplitude of four minutes. Even so, the triple nature of the system was first discovered from spectroscopic observations. Just as in VW Cephei, the true lighttime term would probably have remained masked by much larger random period changes unless other evidence pointed to the necessity of its existence.

$W_{c}$ are only beginning to combine photometric, spectroscopic and visual observations in the study of multiple systems and already we are learning much and finding that gravitationally bound multiple systems are probably a more significant part of the total stellar population than has yet been realized. We believe that further work along these lines is bound to lead to new results that may well prove of basic importance to our understanding of stellar formation and evolution.

Note added in proof.-Since this paper was prepared a much more extensive study of U Ophiuchi has been published by Koch and Koegler (1977). They deduce the parameters of a light-time orbit of period 42 years which is consistent with the available times of minima, but regard such an orbit as tentative. They also assemble the available information on the variations of the phase of the secondary eclipse. In addition some times of secondary minimum have been published by Becker, Chambliss and Kiasat (1975), and these support the relatively late phase of this minimum found in recent years by Eaton and Ward and by the authors.

\section{REFERENCES}

Baize, P. 1959, J. Observateurs, 42, 118.

Bath, G. T., Evans, W. D., and Pringle, J. E. 1974, M.N. R.A.S., 166, 113.

Batten, A. H. 1973, Binary and Multiple Systems of Stars, (Oxford: Pergamon Press) Chap. 2.

Batten, A. H. 1975, Pub. D.A.O., 14, 191.

Batten, A. H., and Hardie, R. H. 1965, A. J., 70, 666.

Batten, A.H., and van Dessel. E. L. 1976, Pub. D.A.O., 14, 345.

Becker, G. A., Chambliss, C. R. and Kiasat, A. 1975. I.A.U. Inf. Bull. Variable Stars, No. 1067.

Berman, L. 1932, Lick Obs. Dall., 16, 24.

Deuerbeck, H.W. 1975, IAU Inf. Bull. Variable Stars, No. 1023.

Deutsch, A.N. 1960, Izv. Glav. Astr. Obs. Pulkove, 22, part 1, No. 166.

Eaton, J.A., and Ward, D.H. 1973, Ap. J., 185, 921.

Ebbighausen, E.G. 1958, Ap. J., 128, 598.

Iiggen, O.J. 1948, $A p . J ., 108,1$.

Evans, D.S. 1968, Quart. J.R.A.S., 9, 388.

Franz, O. G. 1971, Lowell Obs. Bull., No. 154.

Frieboes-Conde, H., and Herczeg, T. 1973, Astron. and Astrophys. Suppl., 12, 1.

Frieboes-Conde, H., Herczeg, T. and $\mathrm{H} \phi \mathrm{g}$, E. 1970, Astron. and Astrophysics, 4, 78.

Gatewood, G., and Eichhorn, H. 1973, A.J., 78, 769.

Harper, W.E., and Blanchet, G.H. 1937, Pub. D.A.O., 7, 99.

Hershey, J. L. 1975, A.J., 80, 662.

Hill, G., Aikman, G. C. L., Cowley, A. P., Bolton, C. T. and Thomas, J. C. 1976, Ap. J., 208, 152.

Irwin, J.B. 1952, $A p$. J., 116, 211.

Irwin, J.B. 1959, A.J., 64, 149.

Knipe, G.F.G. 1971, Pub. A.S.P. 83, 352.

Koch, R. H. and Koegler, C. A. 1977, Ap. J, 214, 423.

Kwee, K.K., and van Woerden, H. 1956, Bull. Astr. Inst. Netherl., 12, 327.

Mancuso, S., and Milano, L. 1975, Ap. and Space Sci., 32, 385. 
McLaughlin, D.B. 1934, Pub. Obs. Univ. Michigan, 6, 3. Morbey, C. L. 1975, Pub. A.S.P., 87, 689.

Petrie, R.M., and Batten, A.H. 1965, Trans. IAU, 12B, 476.

Petrie, R.M., and Batten, A.H. 1969, Pub. D.A.O., 13, 383.

Pohl, E., and Kizilirmak, A. 1974, IAU Inf. Bull. Variable Stars, No. 937.

Scarfe, C.D., Niehaus, R.J., Barlow, D.J., and Baldwin, B.W. 1973, IAU Inf. Bull. Variable Stars, No. 844.
Schöff́el, E., and Popper, D.M. 1974, Pub. A.S.P., 86, 267.

Strand, K. Aa. 1957, A.J., 62, 35.

van de Kamp, P. 1973, A.J., 78, 1099.

van de Kamp, P. 1975, A.J., 80, 658.

van de Kamp, P., Smith, S.F., and Thomas, A. 1950, A.J., $55,251$.

Vetesnik, M. 1968, Bull. Astr. Inst. Czechoslovakia, 19, 123.

West, F. R. 1976, Ap. J., 205, 194.

Worth, M.D., and Heintz, W.D. 1974, Ap. J., 193, 647. 